\title{
A Nonphotic Stimulus Causes Instantaneous Phase Advances of the Light-entrainable Circadian Oscillator of the Syrian Hamster but Does Not Induce the Expression of $c$-fos in the Suprachiasmatic Nuclei
}

\author{
Simon Mead, Francis J. P. Ebling, Elizabeth S. Maywood, Trevor Humby, Joe Herbert, and Michael H. \\ Hastings \\ Department of Anatomy, University of Cambridge, Cambridge CB2 3DY, United Kingdom
}

The study investigated whether nonphotic cues that alter the phase of overt circadian rhythms do so by causing instantaneous shifts in the underlying, light-sensitive clock. Wheel-running activity in Syrian hamsters was studied under free-running conditions of constant dim red light as an overt marker of circadian phase, the daily onset of activity being defined as circadian time 12 (CT 12). Exposure to a $15 \mathrm{~min}$ pulse of bright light at CT $\mathbf{1 2 . 2 0}$ caused a phase delay in activity onset, whereas pulses delivered at CT 11.20 had no effect upon the overt rhythm. Correlated with their effect on behavior, light pulses delivered at CT $\mathbf{1 2 . 2 0}$ induced expression of c-fos-like immunoreactivity in the retinorecipient regions of the suprachiasmatic nuclei of the hypothalamus (SCN), whereas pulses delivered at CT 11.20 had no effect upon the expression of $c$-fos. Expression of this immediateearly gene therefore provided a second marker of circadian phase, because its induction by light is closely correlated with the onset of subjective night (CT 12). To establish a suitable protocol for nonphotic shifts of the activity rhythm, animals were handled and received a subcutaneous injection of saline at different circadian phases. Injections at CT 8 or CT10 caused an immediate bout of wheel-running activity, and a consequent phase advance in the activity rhythm as assessed by the earlier onsets of activity in successive days. Handling and injections at other circadian phases were without effect. Despite shifting the overt rhythm, these procedures at CT 10 did not lead to the expression of $c$-fos in the SCN. One explanation is that increased expression is not a necessary component of all events that shift the clock. Alternatively, although the handling procedures shift the phase of the overt rhythm, they may not instantaneously shift the covert clock. To determine whether, following the nonphotic stimulus, the light-entrainable oscillator had advanced in association with the overt activity rhythm, animals

Received Oct. 8, 1991; revised Jan. 21, 1992; accepted Jan. 23, 1992.

This work was supported by the Medical Research Council, United Kingdom, via Project Grant G8912713N (to M.H.H.). F.J.P.E. was in receipt of an MRC training fellowship. We are extremely grateful to David Hancock and Dr. Gerard Evan, Imperial Cancer Research Fund Laboratories, London, for provision of the antiserum used for immunocytochemistry. John Bashford and colleagues in the Audio Visual Unit, Department of Anatomy, provided excellent assistance with photomicrography, and Kathleen Drea provided excellent assistance with the immunocytochemistry.

Correspondence should be addressed to Dr. Michael H. Hastings, Department of Anatomy, University of Cambridge, Downing Street, Cambridge CR2 3DY, United Kingdom.

Copyright (C) 1992 Society for Neuroscience $0270-6474 / 92 / 122516-07 \$ 05.00 / 0$ that received a saline injection at CT 10.20 were then exposed to either a light pulse or a sham "dark" pulse $1 \mathrm{hr}$ later. In animals that were transferred back to constant dim red light, it was apparent that after injection at CT 10.20, the activity rhythm did not advance as far in animals receiving a subsequent light pulse as it did in controls that received only a dark pulse. The difference in the shift attributable to the light pulse was of the order of $\mathbf{3 0}$ min, equivalent to the delay expected if the animal were exposed to light at CT 12.20. Other animals killed $1 \mathrm{hr}$ after the start of the $15 \mathrm{~min}$ light pulse showed high levels of c-fos expression in the retinorecipient SCN, comparable to that expected following exposure to a light pulse at CT 12.20. These results indicate that when animals received a light pulse $1 \mathrm{hr}$ after a saline Injection at $\mathrm{CT}$ 10.20, the behavioral shift and photoinducibility of $c$-fos were equivalent to those expected of a light presentation after $\mathrm{CT} 12$ and were inconsistent with a phase of $\mathrm{CT} 11.20$. It is therefore concluded that the nonphotic stimulus did phase advance the light-entrainable oscillator of the SCN, as defined by its photic phase-response curve and the photoinducibility of $c$-fos. Furthermore, the absence of $c$-fos following a nonphotic advancing shift demonstrates that despite the correlation between photically induced phase shifts and $c$-fos, enhanced production of this protein within the SCN is not a necessary component of all phase-shifting processes.

The suprachiasmatic nuclei of the hypothalamus (SCN) are the site of the principal circadian oscillator in mammals (Rusak and Zucker, 1979; Ralph et al., 1990; Hastings, 1991). Photic stimuli conveyed directly and indirectly to the nucleus along retinal efferents are responsible for entrainment to the ambient lightdark cycle. Recent studies have also shown that a variety of nonphotic stimuli can also shift free-running circadian rhythms (Aschoff et al., 1971; Mrosovsky 1988, 1989; Mrosovsky et al., 1989). However, it is not clear whether nonphotic entrainment represents a direct effect of the stimulus upon the light-entrainable clock or whether it alters or masks the way in which the endogeneous signal generated by the clock is ultimately expressed, resulting in a new phase angle between the overt rhythm and the covert oscillator that drives it. By examining the behavioral effects of combined photic and nonphotic cues, Mrosovsky (1991) was unable to demonstrate a simple additive or subtractive interaction between the two forms of Zeitgeber, although, as he points out, interpreting such cross-modality studies is complicated because it is never clear whether one stimulus 
alters the salient properties of the other. For example, exposure to light may suppress activity and so attenuate the effect of those cues in which the phase-shifting action is dependent upon increased activity (Mrosovsky, 1991). On the basis of behavioral responses to such double-pulse treatments, Mrosovsky (1991) suggested that such "arousing" (i.e., activity-stimulating) nonphotic stimuli distorted the photic phase-response curve (PRC) and thus led to some form of synergistic interplay between the two stimuli during entrainment. However, the use of activity as the sole marker of circadian phase is likely to confound interpretation, insofar as a change in activity is used not only as a measure of the response to the nonphotic cue, but is also part of the effective stimulus. Therefore, in order to investigate the interaction between photic and nonphotic entrainment further, the present study used the rhythm of photoinducibility of the expression of the immediate-early gene (IEG) $c$-fos within the retinoreceptive zone of the $\mathrm{SCN}$ as a marker of circadian phase. This has several advantages. The effect of light pulses on the expression of $c$-fos in the SCN is very rapid, occurring in less than $1 \mathrm{hr}$, and is correlated with the ability of the pulse to phase shift the free-running clock (Kornhauser et al., 1990; Rusak et al., 1990; Ebling et al., 1991). Light pulses delivered during subjective night that would cause either advances or delays are equally cffective in causing the cxpression of $c$-fos, whereas pulses delivered during subjective day that would not be expected to shift the clock do not induce the expression of this IEG. Consequently, the onset of photoinducibility of expression of $c$-fos can be taken as a marker for the onset of subjective night, independent of overt activity. The expression of IEG also demonstrates unequivocally a change in responsiveness of SCN neurons, thereby identifying directly one component of the neural substrate to any observed phase shift.

A further reason for investigating the effect of nonphotic cues upon the expression of $\mathrm{c}$-fos in the SCN is related to the function of this protein. In common with several other IEGs induced by light pulses, for example, egr-1 (Rusak et al., 1990; Ebling et al., 1991), c-fos is a transcription factor, directing patterns of genomic expression in cells (Sheng and Greenberg, 1990). Several models for the generation of circadian periodicity involve tcmporal programs of genc transcription (Edmunds, 1988; Rosbash and Hall, 1989). In such a scheme, the light-induced expression of IEG may play a causal role in effecting phase shifts by organizing the transcriptional program underlying the clock. Should IEG expression be a necessary component of the internal mechanism of phase shifting the clock, it would be expected to occur in any context where shifts are induced. Alternatively, IEG expression may be a part of the signal transduction pathway by which photic cues gain access to a light-sensitive oscillator, but are not part of the shifting events per se.

In the present study, IEG expression was detected in the form of c-fos-like immunoreactivity (c-fos-ir). To achieve nonphotic shifts of the activity rhythm, animals were given subcutaneous injections of saline at appropriate phases of their activity cycle. Preliminary studies had revealed that these injections and the associated handling of the animal provided a potent nonphotic stimulus to the rhythm of wheel-running activity. Two hypotheses were tested, the first being that enhanced expression of $c$-fos is a necessary component of the mechanisms that direct phase shifts of the SCN. The expression of c-fos-ir was determined in animals following a nonphotically induced shift. If expression of $c$-fos is an integral component of the process that actually shifts the phase of the clock, then any stimulus that shifts the clock would be expected to cause expression of $c$-fos. The second hypothesis was that nonphotically induced shifts of the overt rhythm are not accompanied by shifts in the phase of the underlying, light-entrainable oscillator. The phase of the oscillator after an advancing injection was mapped by describing the response of the activity rhythm and of $c$-fos within the SCN to a light pulse delivered $1 \mathrm{hr}$ after the injection. If the lightcntrainable oscillator did advance immediatcly after a nonphotic cue, then the subsequent light pulse would impinge upon the photosensitive phase of the circadian cycle and so would have parallel effects on both behavioral (phase-shifting) and transcriptional (expression of IEG) end points. If the oscillator did not immediately advance with the overt rhythmicity, then the light pulse would fall at a nonresponsive phase for both measures.

\section{Materials and Methods}

Adult male Syrian hamsters (Wrights of Essex, Chelmsford, United Kingdom) were housed singly in a cage fitted with a running wheel, under a $16 \mathrm{hr}$ light $/ 8 \mathrm{hr}$ dark schedule for 4 weeks prior to experimentation, with food and water available ad libitum. Room lighting was provided by twin fluorescent strip lights of $40 \mathrm{~W}$. A dim red light was on continuously to provide sufficient illumination necessary for manipulations during the dark phase. Wheel running was detected via closure of microswitches and recorded using DATAQUEST III software (Minimitter, Sunriver, OR) running on a computer (Viglen Computers Ltd., London). Actograms were plotted using the corrections described by Mrosovsky and Janik (1991). Light or control dark pulses were provided by transferring animals to an adjacent room and exposing them to white light equivalent to that present during the illuminated phase of the lightdark cycle, or to dim red light equivalent to that used during the dark phase in the home room. Nonphotic phase shifts were achieved by giving animals a subcutaneous injection of $0.5 \%$ ethanolic saline. This involved opening the animal's cage, weighing it on a top pan balance, and then holding the animal steady to inject $100 \mu \mathrm{l}$ of solution at the back of the neck before returning the animal to the cage.

Animals for immunocytochemistry (ICC) received an overdose of pentobarbitone anesthetic (Euthatal, RMB Animal Health, Dagenham, United Kingdom) while under dim red light and were then heparinized and perfused transcardially with PBS followed by $4 \%$ paraformaldehyde in PBS. Brains were removed, postfixed for $2 \mathrm{hr}$, and then transferred overnight to $20 \%$ sucrose in PBS prior to sectioning at $40 \mu \mathrm{m}$ on a freezing microtome. C-fos protein was detected immunocytochemically in free-floating sections using a 1:2000 dilution of an antiserum raised against the $\mathrm{N}$-terminal peptide sequence of the protein (antiserum provided by D. Hancock, Imperial Cancer Research Fund Laboratories, St. Bartholomew's Hospital, London). Primary antibody was detected using a Vectastain avidin-biotin complex kit with diaminobenzidine visualization. This procedure has been described in greater detail previously (Ebling et al., 1991). C-fos expression in the SCN was quantified by counting immunopositive nuclei. Digitized images were captured via a black-and-white video camera attached to a Zeiss microscope (Seescan, Cambridge, United Kingdom). The number of c-fos-immunopositive nuclei in the SCN was counted, bilaterally, in each of the two adjacent coronal sections from the caudal region of the nuclei that contained the greatest amount of staining. The mean count per section was calculated for each animal, and the final results were expressed as the overall group mean $( \pm$ SEM) of the individual animal means.

\section{Protocol}

Experiment I. The effect of light pulses encountered at around C'T 12 on phase shifts of the activity rhythm and the expression of $c$-fos-ir within the $S C N$. Animals were released into constant dim red light and allowed to free-run for approximately $10 \mathrm{~d}$. Separate groups of animals were then exposed to a 15 min light pulse at either circadian time (CT) 11.20 or CT 12.20 , CT 12 being anticipated onset of activity. Half of the animals in each group were killed $1 \mathrm{hr}$ after the start of the light pulse and processed for ICC ( $n=9$ for CT $11.20 ; n=9$ for CT 12.20). Activity recording continued for the remaining animals to determine whether the pulse caused a phase shift in the activity rhythm $(n=7$ for CT $11.20 ; n=10$ for CT 12.20). A further group of animals were held on 


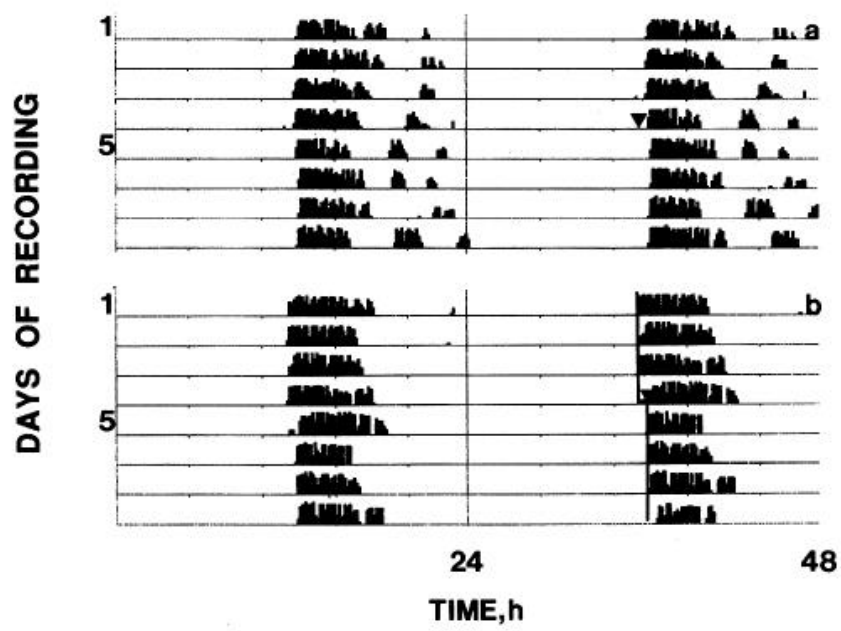

Figure 1. Representative double-plotted traces of wheel-running activity of hamsters held in constant dim red light and exposed to a light pulse for $15 \mathrm{~min}$ at CT $11.20(a)$ or CT $12.20(b)$, where CT 12 is defined as the activity onset prior to treatment. Arrowhead denotes start of light pulse. Lines on right side in the lower actogram indicate the activity onsets, fitted by eye, that were used to calculate the phase shift.

dim red light and exposed to sham "dark" pulses by being transferred between rooms with dim red illumination at CT $11.20(n=3)$ or CT $12.20(n=3)$ and then processed for ICC $1 \mathrm{hr}$ later.

Experiment 2. The effect of "arousal," induced by the injection of saline, upon the free-running activity rhythm and the expression of $c$-fos-ir within the SCN. To determine whether the expression of c-fosir is an inevitable consequence of phase shifts of the circadian activity rhythm, animals were allowed to free-run for approximately $10 \mathrm{~d}$ and were then given a saline injection at CT $2,6,8,10,14,20$, or 23 to determine the most sensitive phase for nonphotic shifts $(n=4$ for all points except CT 10 , where $n=17$ ). They were then returned to their home cage and their activity recorded for at least $6 \mathrm{~d}$ in order to determine the magnitude of any subsequent phase shift. Some animals that had exhibited a shift at CT 10 received a second injection at CT 10 several days later to determine whether sensitivity to the nonphotic stimulus became attenuated with repeated presentation. Other animals that had earlier showed a response to injection at CT 10 were injected at CT 10 a second time and were then killed and processed for ICC 1 hr later $(n=4)$.

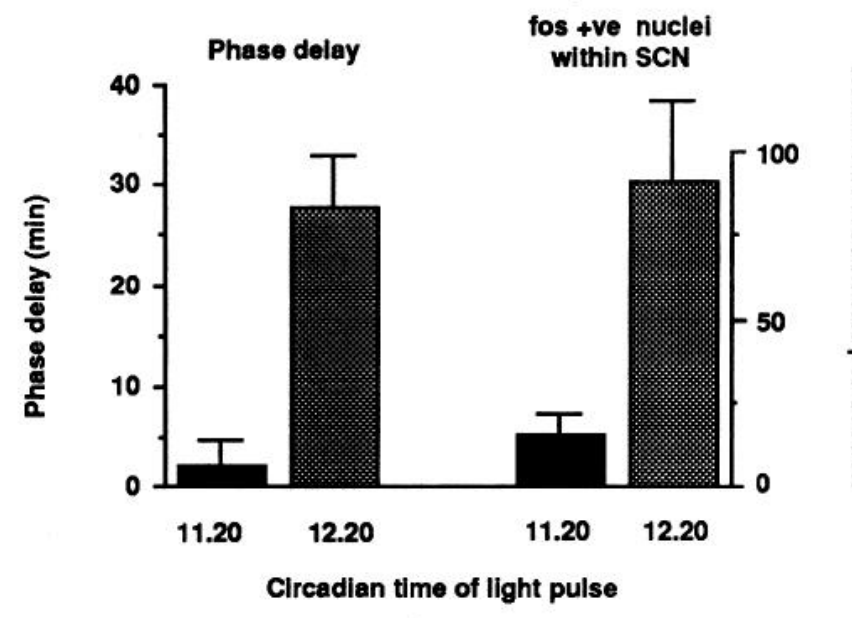

Figure 2. Magnitude of phase delays and counts of c-fos-ir nuclei within the SCN (means \pm SEM) of free-running hamsters pulsed with light at CT 11.20 or CT 12.20 . Group mean numbers of c-fos-positive nuclei are calculated from the means of the two sections with the highest cell count for each individual.
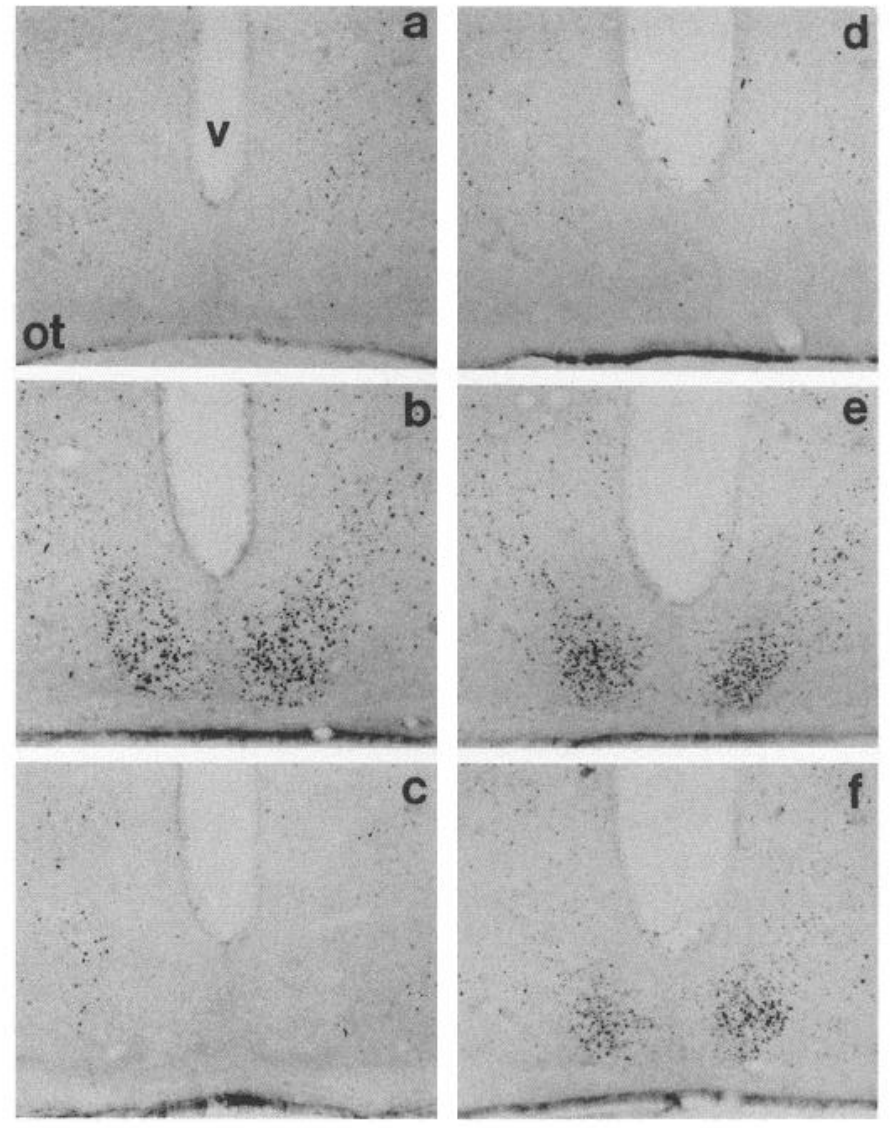

Figure 3. Photomicrographs of coronal sections of the SCN of hamsters held on constant dim red, processed for c-fos-ir. $v$, third ventricle; $o t$, optic tract. Treatments were as follows: $a, 15 \mathrm{~min}$ light pulse at CT $11.20 ; b, 15$ min light pulse at CT 12.20; $c$, saline injection at CT 10; $d$, saline injection at CT 10.20 , followed by dark pulse $1 \mathrm{hr}$ later; $e$ and $f$, saline injection at CT 10.20 , followed by a $15 \mathrm{~min}$ light pulse $1 \mathrm{hr}$ later.

Experiment 3. The effect of nonphotic phase shifts upon the phase of the light-entrainable oscillator of the SCN. The aim of this experiment was to determine whether the advances in the activity rhythm caused by presentation of the nonphotic stimulus at CT 10 were accompanied by a comparable advance in the phase of the light-entrainable oscillator. Animals held under free-running conditions for $10 \mathrm{~d}$ or more received a saline injection at CT 10.20 . Half of them then received a $15 \mathrm{~min}$ light pulse $1 \mathrm{hr}$ later, whereas the remainder received a control "dark" pulse. Some of the animals were then allowed to continue their freerun for several days to determine the magnitude of any phase shifts resulting from the treatment (light pulse, $n=7$; "dark" pulse, $n=7$ ). The remainder were killed $1 \mathrm{hr}$ after the start of the light pulse $(n=6)$ or dark pulse $(n=7)$ and processed for ICC. The rationale behind the experiment was that if the injection at CT 10.20 did cause an instantaneous advance of the underlying light-entrainable oscillator of approximately $1 \mathrm{hr}$, the light pulse falling $1 \mathrm{hr}$ later (external time) would be encountered not at CT 11.20 but at CT 12.20. It would therefore fall into the phase for photoinduction of c-fos-ir within the SCN and the phase at which light would cause a phase delay of the overt activity rhythm. If the underlying clock did not instantaneously advance following the nonphotic stimulus, then the light pulse would fall in the nonresponsive phase of CT 11.20 and so would be ineffective on either measure. As a control procedure, a separate group of animals received four saline injections at 15 min intervals between CT 8 and CT 9 and were held in the light for that hour, before being killed for ICC. This was to test whether the combination of injection, enforced activity (due to repeated disturbance), and light inevitably caused the expression of c-fos-ir, regardless of the circadian phase of treatment.

Differences between treatment groups were assessed by analysis of variance and post hoc Dunnett's $t$ test. 


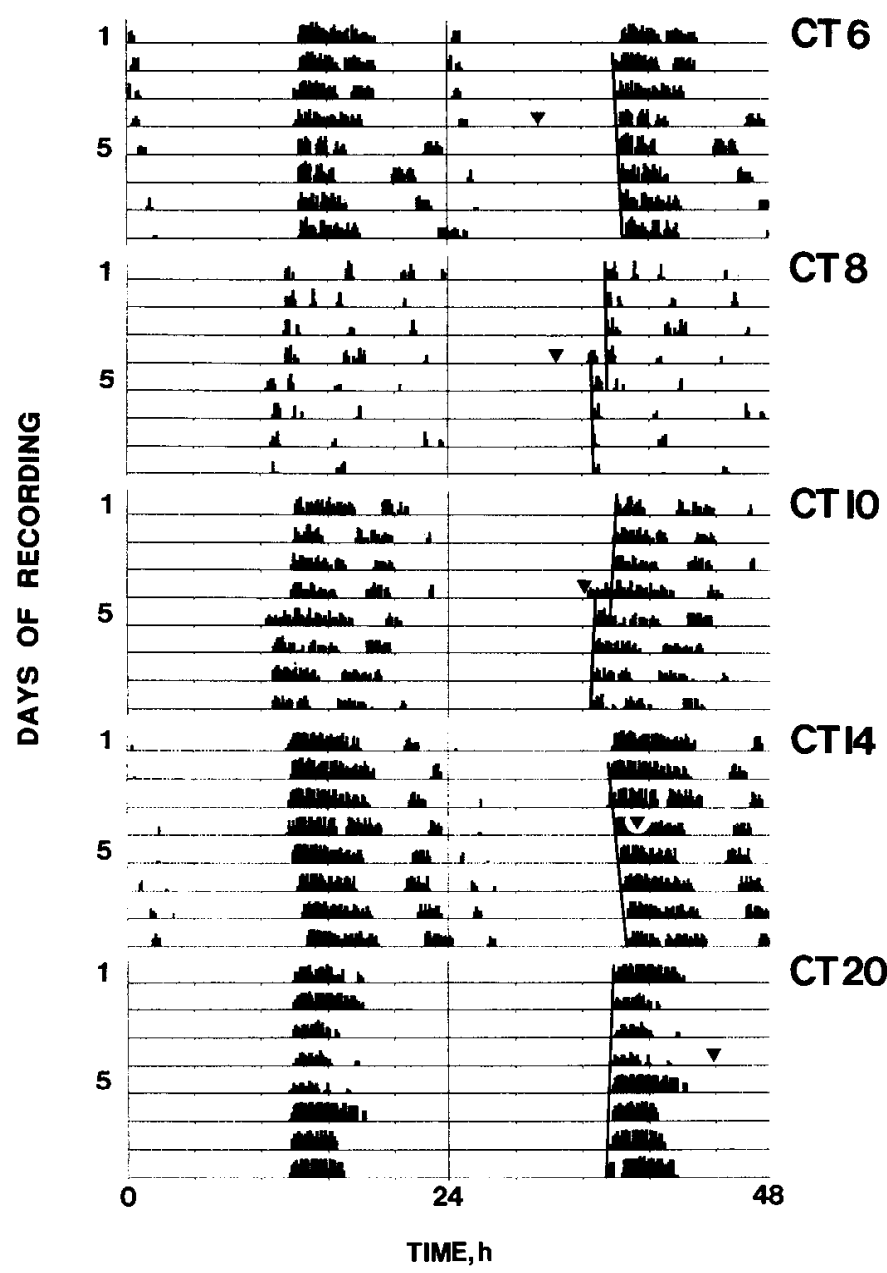

Figure 4. Representative double-plotted traces of wheel-running activity of hamsters held in constant dim red light and injected with saline at various circadian phases. Lines on right side indicate activity onsets for animals, fitted by eye, that were used to calculate CT 12 prior to treatment, and the subsequent phase shifts. Arrowheads indicate time of injection.

\section{Results}

Experiment 1. The effect of light pulses encountered at around CT 12 on phase shifts of the activity rhythm and the expression of $c$-fos-ir within the $S C N$

Exposure of free-running animals to a light pulse at CT 11.20 had no effect on the activity rhythm (Fig. 1a), whereas a pulsc delivered at CT 12.20 caused a phase delay (Fig. 1b) of approximately $30 \mathrm{~min}$ (Fig. 2). In control animals not exposed to light, cell counts of c-fos-ir within the SCN at both CT 11.20 and CT 12.20 were very low $(<20)$. Photic induction of c-fosir within the SCN correlated with the effect of the light pulse upon behavior. Whereas a light pulse at CT 11.20 had no effect on c-fos-ir, a pulse $1 \mathrm{hr}$ later caused extensive expression of c-fos-ir throughout the retinorecipient regions of the SCN (Fig. $3 a, b)$. Cell counts revealed that the mean number of c-fospositive nuclei in animals pulsed at CT 11.20 was less than 25 (Fig. 2). In contrast, a pulse at CT 12.20 caused a mean count greater than 90 . Of eight animals tested at CT 11.20 , only one showed appreciable counts $(>30)$ of c-fos-ir nuclei, whereas only one of eight pulsed at CT 12.20 failed to exhibit this number of counts. These results confirm the temporal correlation be-

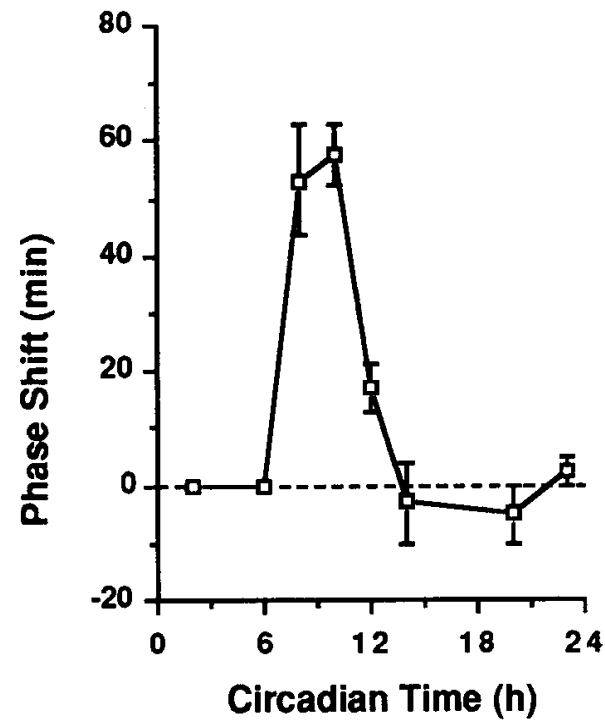

Figure 5. PRC to the injection of saline. Symbols indicate group mean $+\operatorname{SEM}(n=4$ per group, except at CT 10,n=17). Where error bars are absent, value is within the symbol.

tween the phase-shifting effect of a light pulse and the photoinducibility of $c$-fos-ir within the SCN. Moreover, they show that the onset of photoinducibility is sufficiently robust and precise to be used as a marker for the onset of subjective night.

Experiment 2. The effect of "arousal." induced by the injection of saline, upon the free-running activity rhythm and the expression of $c$-fos-ir within the $S C N$

Injection of saline at CT 2, 6, 14, 20, or 23 had no effect upon free-running activity rhythms (Figs. 4,5 ). However, when given at CT 8 or CT 10, the injection procedure was followed by an earlier than anticpated bout of wheel-running activity, and over subsequent circadian cycles a marked phase advance in activity onset was apparent. In the case of CT 10, the earlier activity followed immediately after injection, whereas with most animals injected at CT 8 , the earlier activity did not commence until some time after the animal was returned to its cage after the injection. The advancing effect of injection at CT 10 was not attenuated for a second injection given several days later (first injection, $+60 \pm 8 \mathrm{~min}$, vs. second, $+56 \pm 8 \mathrm{~min}, n=$ 9). The PRC to saline injection is presented in Figure 5, which shows that the mean advance following injection at CT 10 was approximately $60 \mathrm{~min}$. Overall, there was a highly significant effect of time on the phase-shifting response to injection $(F=$ $16.5 ; \mathrm{df}=7,4 ; p<0.01)$. All animals processed for ICC showed an immediate behavioral response to the saline injection (Fig. 6), and thus a shift in their circadian timing would have been anticipated. However, examination of the SCN of these animals showed that there was no induction of c-fos-ir within the SCN $1 \mathrm{hr}$ after treatment, all animals exhibiting a count of less than 10 nuclei. A section through the caudal SCN from a representative animal is shown in Figure $3 c$.

\section{Experiment 3. The effect of nonphotic phase shifts upon the} phase of the light-entrainable oscillator of the SCN

Animals that received an injection of saline at CT 10.20 followed by a dark pulse $1 \mathrm{hr}$ later showed the anticipated phase 


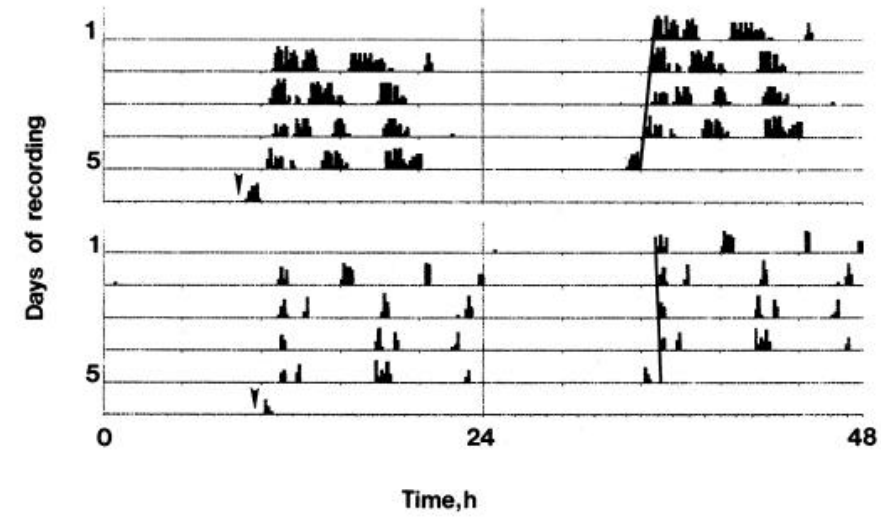

Figure 6. Representative double-plotted traces of wheel-running activity of two hamsters held under constant dim red light and injected with saline at CT 10 (arrowheads). Lines on right side indicate activity onsets for animals fitted by eye, that were used to define CT 12. The hamsters were perfused for c-fos ICC at CT 11 .

advance in the rhythm of wheel running (Fig. 7a,b), the magnitude of which was approximately $40 \mathrm{~min}$, slightly less than that observed in animals injected at CT 10.00 precisely (Fig. 5). Animals that received a light pulse $1 \mathrm{hr}$ after the injection showed a much smaller advancing shift (Fig. $7 c, d$ ), with a group mean of around $10 \mathrm{~min}$ (Fig. 8). The difference in the magnitude of the advances exhibited by the two groups, and which was therefore attributable to the light pulse, was of the order of $30 \mathrm{~min}$, equivalent to the expected delay if the light pulse fell at CT 12.20 (see Fig. 2).

Animals that were injected with saline at CT 10.20 and were then exposed to a dark pulse did not exhibit detectable amounts of c-fos-ir within the SCN (Fig. 3d). The mean count of c-fospositive nuclei in this group was $3.6 \pm 1.7$. However, all of the animals that were exposed to the $15 \mathrm{~min}$ light pulse $1 \mathrm{hr}$ after injection showed extensive immunoreactivity in the typical pattern of retinoreceptive portions of the nucleus (Fig. $3 e, f$ ), the mean count for the group being $129 \pm 22$ nuclei per section. Animals in the control group that received multiple injections and exposure to light for $1 \mathrm{hr}$ during the interval between CT 8 and CT 9 exhibited negligible c-fos-ir in the $\mathrm{SCN}$ ( $<10$ counts), confirming the temporal specificity of the effect after injection at CT 10.20. This is consistent with an advancing shift of the light-entrainable clock following the nonphotic stimulus.

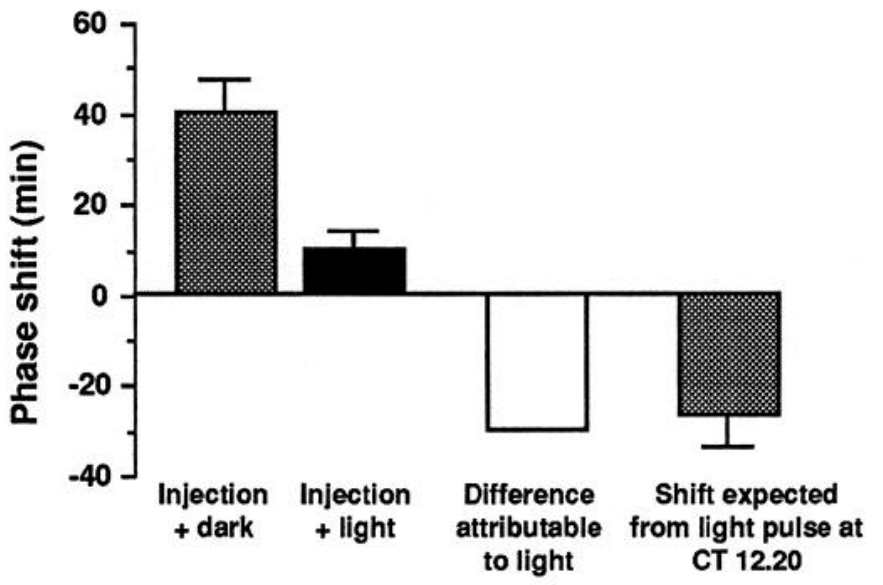

Figure 8. Mean ( \pm SEM) phase shifts in wheel-running activity following injection of saline at CT 10.20 , combined with a "dark" or a light pulse $1 \mathrm{hr}$ later.

\section{Discussion}

The onset to the photoinducible phase for $c$-fos expression in the $\mathrm{SCN}$ is very precisely synchronized (within less than $1 \mathrm{hr}$ ) to the onset of subjective night. This phase is associated with the start of the delay portion of the photic PRC. The free-running circadian rhythm of activity can also be phase advanced by a nonphotic stimulus, a single subcutaneous injection of saline, applied before activity onset, but such nonphotic shifts are not associated with the enhanced expression of $c$-fos within the SCN. This result demonstrates that the enhanced expression of $c$-fos is not an integral and necessary component of all procedures that shift circadian timing. However, by the combined use of saline injections followed by the presentation of a light pulse to probe for the phase of the light-sensitive oscillator, it was possible to demonstrate that nonphotic cues do cause an instantaneous $(<1 \mathrm{hr})$ phase advance of the light-sensitive oscillator, since the response to subsequent photic stimuli is altered. It is clear that at least two different modes of Zeitgeber, photic and nonphotic, exert an influence over the phase of the SCN oscillator, and they achieve this by two separable routes, insofar as photic entrainment is correlated with the induction of c-fos-ir whereas nonphotic cues shift the clock by different means.

The anatomical distribution of c-fos-ir following presentation
Figure 7. Representative doubleplotted traces of wheel-running activity of hamsters held under constant dim red light and injected with saline at CT 10.20 (arrowheads), which were subsequently exposed to a "dark" pulse ( $a$, $b$, solid circle) $1 \mathrm{hr}$ after the injection, or exposed to a $15 \mathrm{~min}$ light pulse $(c$, $d$, star within circle), $1 \mathrm{hr}$ after the injection.
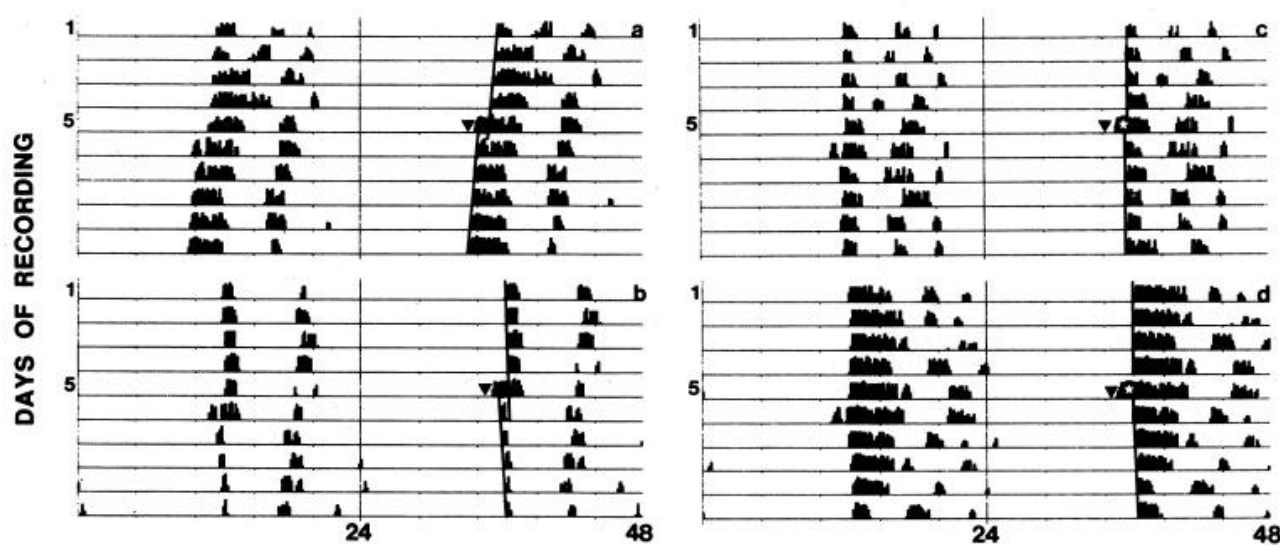

TIME, $\mathrm{h}$ 
of a light pulse during early subjective night was comparable to that described previously in rodent species (Kornhauser et al., 1990; Rusak et al., 1990; Ebling et al., 1991), being restricted to the retinorecipient zones of the nucleus (Pickard, 1982). These earlier studies showed that the photoinduction of IEG expression was temporally as wcll as spatially specific, occurring only after light pulses delivered during subjective night. The present results provide greater resolution to this finding and show that the photoinducible phase is precisely regulated within the circadian cycle with an accuracy of at least $1 \mathrm{hr}$. As such, the onset of the photoinduction of $c$-fos was a useful marker for the instantaneous determination of one phase of the light-entrainable oscillator. However, other studies have revealed that the pattern of $c$-fos induction resulting from exposure to light at other phases of subjective night does not exhibit any predictable variation, and so the utility of the marker is restricted solely to determining the onset, and possibly the offset, of subjective night, rather than to determining particular delay or advance phases of the PRC.

The phase-dependent phase shift of the activity rhythm following the injection of saline shared many of the properties described for other nonphotic cues. The effects of such a stimulus on arousal following handling and injection and the subscquent induction of locomotor activity may be particularly important (Turek, 1989). Increased locomotor activity is commonly observed following presentation of many other nonphotic Zeitgebers, although the physiological or neural consequences of this increased activity that might ultimately influence the clock are not yet known (Mrosovsky, 1988, 1989, 1991; Mrosovsky et al., 1989; Van Reeth and Turek, 1989; Mrosovsky and Salmon, 1990). It should be noted that the shape of the nonphotic (injection) PRC was different from other nonphotic curves: the injection stimulus failed to produce any evidence of phase delays, and the sensitive phase for advances was restricted to a much later portion of subjective day than has been reported for stimuli such as induced wheel running in a treadmill, treatment with the benzodiazepine drug Triazolam, or transfer to a novel environment. However, although nonphotic stimuli may be applied at particular circadian times, it is not nccessarily the casc that they have their effects upon the clock only at that time. For example, the physiological consequences of drug administration, induced exercise, or olfactory excitement may persist some time after the proximate stimulus is terminated. If these consequential changes are the important factor in entrainment, they may impinge upon a later, sensitive phase of the cycle and exert their effects at that point. Consequently, where different treatments have different time courses to their immediate and longerterm physiological effects, they may yield apparently different PRCs, even though ultimately they are acting upon the clock through the same mechanism at the same clock phase. As a result, it will be difficult to equate and compare manipulations exploiting different modalities until the critical parameters of nonphotic shifts are better characterized.

A second feature raised by these studies is the potent effect of a presumed "control" procedure, the injection of ethanolic saline, which is a commonly used vehicle. This inevitably raises the questions of how robust a Zeitgeber this stimulus actually is, and why is it not more widely reported in studies using pharmacological manipulations of the free-running clock. One possibility is that repeated injection would habituate the animal to the arousing effects of handling and injection and so entrainment would become attenuated. In the present work, animals received no more than three injections, separated by several days of isolation, and so habituation would be less likely to occur. Nevertheless, it is clear from some studies in rats that saline injections can indeed shift the circadian activity rhythm when delivered during late subjective day (Armstrong, 1989), although given the narrow window of sensitivity, such effects may often be overlooked in more extensive phase-response studies where experimental, but not control, groups exhibit shifts at other phases of the cycle.

The aim of the final experiment was to determine whether or not the nonphotic stimulus advanced the phase of the lightentrainable oscillator, as indicated by the phase of photoinducibility of c-fos-ir and the delaying response to light. The results suggest that this was the case. An injection at CT 10.20 resulted in the subsequent light stimulus giving a shift in activity and inducing the expression of $c$-fos as if the clock had been advanced so that the light pulse delivered $1 \mathrm{hr}$ later actually fell at CT 12.20. The additive effect of double light pulses applied to a free-running photosensitive clock has been demonstrated previously for the pupal eclosion rhythm of Drosophila pseudoobscura (Pittendrigh, 1974), but there is currently no theoretical basis within which to predict the interaction between Zeitgebers of differcnt modalitics. In the study by Mrosovsky (1991), the combined effect of an advancing nonphotic stimulus and a subsequent light pulse applied to one of a series of individual phases could not be explained as a simple summation, although qualitatively additive and subtractive effects did occur and appeared to follow in general outline the anticipated phase of the lightentrainable PRC. The present study did not pursue exhaustively the position of the full photic PRC. In principle, this would have been possible by delivering light pulses to different groups at different phases, thereby to map the PRC by inferring its position from subsequent shifts in the activity rhythm. In the light of the findings of Mrosovsky (1991), this approach may become tautological, insofar as the shifts and phase of the clock are used to predict and verify each other. In contrast to Mrosovsky's conclusions, the present data based both upon overt activity and upon the photoinducibility of $c$-fos in the SCN, and on the basis of the single phase studied, do fit well with a model (Mrosovsky, 1991) in which the nonphotic cue causes an advancing shift in the entire photic PRC. Reconciliation of the two conclusions is difficult, not least because the two studies used contrasting experimental protocols, particularly the nature of the nonphotic cues, and the use by Mrosovsky of the Aschoff type 2 experiment in which animals were held under a light-dark cycle and released into constant conditions only at the lime of treatment. In contrast, the present study employed the more conventional procedure of using animals that had been allowed to free-run for some time before being presented with a Zeitgeber pulse. This means that confounding influences such as decompression of the nocturnal activity bout that occurs upon release into constant dim light, and potential differences in pre- and post-pulse period, can be discounted. Whether such procedural differences alone are able to explain the differences observed seems unlikely, but such a problem is difficult to reconcile when activity is the sole assay for clock phase.

Regardless of the precise mechanisms involved, the present observation that the nonphotic stimulus advances the phase of photoinduction of IEG supports the general conclusion drawn by the behavioral studies reported here and by Mrosovsky (1991), that nonphotic cues shift the circadian rhythm of photic sensitivity. The clear demonstration of an effect within the SCN reported in the present study indicates that nonphotic cues have 
their effect by impinging upon the activity of this nucleus, rather than by modifying the expression of an essentially unaltered SCN signal. However, although it is concluded that the lightentrainable oscillator is phase shifted by nonphotic stimuli, the level of interaction between the two pathways is not known. The light-mediated phase shifts and the photic induction of expression of IEG in the SCN are probably controlled, in part, by glutamatergic retinal efferents. Treatment of animals with antagonists to the NMDA subtype of glutamate receptor blocks light-induced phase shifts (Colwell et al., 1990; Lord et al., 1990) and partially blocks the photic induction of expression of c-fosir within the retinorecipient SCN (Ebling et al., 1991). However, the neurochemical and neuroanatomical mediation of nonphotic entrainment remains an open question. It has been suggested that a second oscillator, separate from the SCN but functionally coupled to it, is sensitive to "arousing" stimuli (Rosenwasser and Adler, 1986; Mrosovsky, 1988). If this were the case, the effects of "arousal" upon the SCN rhythm would be mediated by an indirect mechanism. In view of the fact that all nonphotic cues appear to exert their effect through "arousal," it is very likely that ascending brainstem reticular systems may be involved. In particular, ascending aminergic ncurons, cither noradrenergic, serotoninergic, or dopaminergic, could be part of the indirect pathway (Everitt et al., 1990). Whether nonphotic entrainment can occur in the absence of these pathways, and whether these neurons impinge directly upon SCN neurons or act via a second neural component are questions that await further experimentation.

\section{References}

Armstrong SM (1989) Melatonin and circadian control in mammals. Experientia 45:932-938.

Aschoff J, Fatranoska M, Giedke F (1971) Human circadian rhythms in constant darkness, entrainment by social cues. Science 171:213215.

Colwell CS, Ralph MR, Menaker M (1990) Do NMDA receptors mediate the effects of light on circadian behavior? Brain Res 523: 117-120.

Ebling FJP, Maywood ES, Staley K, Humby T, Hancock DC, Waters CM, Evan GI, Hastings MH (1991) The role of NMDA-type glutamatergic neurotransmission in the photic induction of immediate early gene expression in the suprachiasmatic nuclei of the Syrian hamster. J Neuroendocrinol 3:641-652.

Edmunds LN (1988) Cellular and molecular bases of biological clocks: models and mechanisms for circadian timing. New York: Springer.

Everitt BJ, Robbins TW, Selden NRW (1990) Functions of the locus ceruleus noradrenergic system: a neurobiological and behavioural synthesis. In: The pharmacology of noradrenaline in the CNS (Heal D, Marsden C, eds), pp 349-378. Oxford: Oxford UP.

Hastings MH (1991) Neuroendocrine rhythms. Pharmacacol Ther 50: 35-71.

Kornhauser JM, Nelson DE, Mayo KE, Takahashi JS (1990) Photic and circadian regulation of $c$-fos gene expression in the hamster suprachiasmatic nucleus. Neuron 5:127-134.

Lord AB, Turek FW, Takahashi JS (1990) Application of MK-801 into the suprachiasmatic region attenuates the phase-shifting effects of light on the circadian activity rhythm of the hamster. Soc Neurosci Abstr 16:317.16.

Mrosovsky N (1988) Phase response curves for social entrainment. J Comp Physiol 162:35-46.

Mrosovsky N (1989) Nonphotic enhancement of adjustment to new light dark cycles: masking interpretation discounted. J Biol Rhythms 4:365-370.

Mrosovsky N (1991) Double-pulse experiments with nonphotic and photic phase-shifting stimuli. J Biol Rhythms 6:167-179.

Mrosovsky N, Janik D (1991) Technical comment: in quest of data. J Biol Rhythms 6:93-95.

Mrosovsky N, Salmon PA (1990) Triazolam and phase-shifting acceleration re-evaluated. Chronobiol Int 7:35-41.

Mrosovsky N, Reebs SG, Honrado GI, Salmon PA (1989) Behavioural entrainment of circadian rhythms. Experientia 45:696-702.

Pickard GE (1982) The afferent connections of the suprachiasmatic nucleus of the golden hamster with emphasis on the retinohypothalamic projection. J Comp Neurol 211:65-83.

Pittendrigh CS (1974) Circadian organization in cells and the circadian organization of multicellular systems. In: The neurosciences: third study program (Schmitt FO, Worden FG, eds), pp 437-458. Cambridge, MA: MIT Press.

Ralph MR, Foster RG, Davies FC, Menaker M (1990) Transplanted suprachiasmatic nucleus determines circadian period. Science 247: 975-978.

Rosbash M, Hall JC (1989) The molecular biology of circadian rhythms. Neuron 3:387-398.

Rosenwasser AM, Adler NT (1986) Structure and function in circadian timing systems: evidence for multiple coupled circadian oscillators. Neurosci Biobehav Rev 10:431-448.

Rusak B, Zucker I (1979) The neural regulation of circadian rhythms. Physiol Rev 59:449-526.

Rusak B, Robertson HA, Wisden W, Hunt SP (1990) Light pulses that shift rhythms induce gene expression in the suprachiasmatic nucleus. Science 248:1237-1240.

Sheng M, Greenberg ME (1990) The regulation and function of $c$-fos and other immediate early genes in the nervous system. Neuron 4: 477-485.

Turek FW (1989) Effects of stimulated physical activity on the circadian pacemaker of vertebrates. J Biol Rhythms 4:135-147.

Van Reeth O, Turek FW (1989) Stimulated activity mediates phase shifts in the hamster circadian clock induced by dark pulses or benzodiazepines. Nature 339:49-51. 